Research Article

\title{
Experimental Investigation on Drying and Wetting of GMZ Bentonite Using X-Ray Tomography and Volumetric Digital Image Correlation Technique
}

\author{
Liufeng Chen, ${ }^{1}$ Kang $\mathrm{Li}^{2}{ }^{2}$ and Diansen Yang $\mathbb{D}^{2}$ \\ ${ }^{1}$ College of Civil Engineering, Suzhou University of Science and Technology, Suzhou 215011, China \\ ${ }^{2}$ State Key Laboratory of Geomechanics and Geotechnical Engineering, Institute of Rock and Soil Mechanics, \\ Chinese Academy of Science, Wuhan 430071, China \\ Correspondence should be addressed to Diansen Yang; dsyang@whrsm.ac.cn
}

Received 25 October 2020; Revised 29 November 2020; Accepted 30 November 2020; Published 9 December 2020

Academic Editor: Pingye Guo

Copyright $(2020$ Liufeng Chen et al. This is an open access article distributed under the Creative Commons Attribution License, which permits unrestricted use, distribution, and reproduction in any medium, provided the original work is properly cited.

To investigate the shrinking and swelling properties of Gao-Miao-Zi (GMZ) bentonite, which has been considered as engineering barriers for high-level radioactive nuclear waste disposal in China, drying and wetting tests were carried out. The microstructure of the material at each hydric state was recorded using X-ray tomography (X-CT). The 3D images of the material were analyzed using digital volume correlation (DVC) technique, and the full-field strains at mesoscale (i.e., in the order of clay aggregate) during drying and wetting were quantified. The results show that the GMZ bentonite exhibits notable swelling characteristics during wetting and the swelling strain of the material is up to $8 \%$ at mesoscale. The full-field strain in $3 \mathrm{D}$ of the material is heterogeneous, anisotropic, and irreversible during drying and wetting. The corresponding deformation mechanisms are discussed. Two different swelling mechanisms were also identified: traction occurs in the interface between the matrix and inclusions during swelling; preexisting fissure closes during swelling.

\section{Introduction}

Bentonite clay is often chosen as a buffer and backfill material between the waste canister and the host rock or as seals in excavated disposal galleries in geological disposal of highlevel radioactive waste. It exhibits a few favorite characteristics, such as high swelling, strong sorption ability, and low hydraulic conductivity [1-4].

A better understanding of how the barriers evolve with time is required to license a final repository for high-level radioactive waste. This understanding is based on scientific knowledge about the boundary conditions and processes acting on the barriers in the repository. The processes often include thermal, hydraulic, biochemical, and mechanical processes. For example, local groundwater will flow to the repository once the repository is closed, and bentonite will absorb water and swell. The swelling ability can reduce macroscopic porosity to limit microbial activity, reduce the hydraulic conductivity, prevent any further propagation of the excavation disturbed zone (EDZ) in the rock, and ensure that the buffer is self-sealing. Therefore, understand the swelling process is critical in the evaluation of the long-term performance of a bentonite buffer [3-8].

The shrinking and swelling properties of clayey materials (e.g., argillite and bentonite) have been extensively investigated at various scales using different methods $[9,10]$. Mercury intrusion porosimetry (MIP) is often used to quantify the pore size distribution of the material at different hydrated states [11, 12]. Scanning electron microscopy (SEM) and X-ray tomography (X-CT) are often used to study the microstructure of the material [13-17], and these studies are rather qualitative than quantitative [18]. In recent years, digital volume correlation (DVC) technique is used to obtain the full-field strain of the material and quantify the volume change of materials during hydro-mechanical tests [18-21]. These studies focus on localized deformation of 
materials during different loadings. However, there are few studies focusing the swelling and shrinkage of bentonite during wetting and drying cycles, combining X-CT and DVC techniques.

In this paper, the swelling and shrinkage of the GaoMiao-Zi (GMZ) bentonite, considered as a possible material for engineered barriers in Chinese geological disposal $[1,3,4,8]$, are studied. We present a new procedure to conduct drying and wetting tests combined with X-CT measurements. The shrinking and swelling properties of the GMZ bentonite at mesoscale are quantified and analyzed by digital volume correlation technique. The measured full-field strain of the GMZ bentonite in 3D allows to follow the evolution of damage. The deformation mechanisms of the material during wetting and drying are revealed by analyzing the microstructure changes.

\section{Materials and Methods}

2.1. Materials. The GMZ bentonite from the northern Chinese Inner Mongolia, an autonomous region $300 \mathrm{~km}$ northwest of Beijing, was used for the study. The GMZ bentonite is a sodium bentonite. The mineral composition and physical parameters of the GMZ bentonite is listed in Table 1. It is composed of montmorillonite (75.4\%), quartz (11.7\%), feldspar (4.3\%), and cristobalite (7.3\%) [1]. It has a liquid limit of $276 \%$ and a plastic limit of $37 \%$.

\subsection{Sample Preparation. The GMZ bentonite was crushed} into fine powders, and most of the particles had sizes smaller than $0.01 \mathrm{~mm}$. The GMZ bentonite powder material was preconditioned in an environmental test chamber at $90 \%$ relative humidity and $20^{\circ} \mathrm{C}$ until a constant mass was reached. The GMZ specimen was prepared by compacting the treated powders in a rigid oedometer cell with an inner diameter of $50 \mathrm{~mm}$. The dry density of the prepared specimen is $1.72 \mathrm{~g} / \mathrm{cm}^{3}$, and the initial water content is $15.16 \%$. The earlier studies $[3,8]$ show that the compacted GMZ bentonite has low permeability due to its small pore size.

To obtain the shorter testing duration of the compacted GMZ bentonite and record high 3D imaging resolution, two smaller samples with a diameter of $4 \mathrm{~mm}$ and about a length of $8 \mathrm{~mm}$ were cored from the compacted GMZ specimen, as shown in Figure 1. The axe of sample No. 1 is parallel to the compaction direction, and the axe of sample No. 2 is in the plane perpendicular to the compaction direction.

To avoid being damaged and desaturated, samples No. 1 and No. 2 were placed in a plastic tube with a hole (Figure 2(b)). The water vapour under different constant relative humidity conditions then enters the tube through the hole. The changed relative humidity will saturate or desaturate the sample during the drying and wetting tests.

2.3. Experimental Methods. When the sample reached the hydrated equilibrium state under which the sample weight remained constant, the hole was sealed with a rubber stopper, the sample in the tube was placed in the micro-CT machine (ZEISS Xradia 410 Versa) (Figure 2), and the 3D
TABle 1: Characteristics of the GMZ bentonite (Liu et al., 2001).

\begin{tabular}{lc}
\hline Properties & Value \\
\hline Contents of montmorillonite (\%) & 75.4 \\
Contents of quartz (\%) & 11.7 \\
Contents of feldspar (\%) & 4.3 \\
Contents of cristobalite (\%) & 7.3 \\
Specific gravity & 2.66 \\
Liquid limit (\%) & 276 \\
Plastic limit (\%) & 37 \\
Specific surface area $\left(\mathrm{m}^{2} / \mathrm{g}\right)$ & 597 \\
\hline
\end{tabular}

microstructure at each hydrated state was obtained by recording X-ray radiographic images of the material at several different angular positions. When the scanning of the sample microstructure was completed, the sample was replaced in the container to perform another hydrated test (Figure 2(b)). Note that, during scanning, the state of the sample was considered stable as it was isolated from external environment and the temperature was kept at $25^{\circ} \mathrm{C}$, and the measured weight of the sample was constant before and after $\mathrm{X}$-CT measurement. In this study, the samples were first wetted under the relative humidity (RH) of $98 \%$. The equilibrium state at $\mathrm{RH}=98 \%$ is considered as the initial state. The drying and wetting tests were carried out by changing $\mathrm{RH}$ from $98 \%$ to $75 \%, 58 \%, 32 \%, 58 \%, 75 \%$, and $98 \%$, progressively. The relative humidity in the container was controlled using different supersaturated saline solutions, which are widely used by many researchers $[6,7,11]$. Note that almost all of the dehydration or hydration tests lasted three days to reach the hydrated equilibrium state.

2.4. Experimental Data Processing. The recorded virtual slices are reconstructed using iterative algorithm, and the reconstructions of the CT images have a voxel size of $5 \times 5 \times 5 \mu \mathrm{m}^{3}$, which is in the order of clay aggregates. To quantify the hydrated deformation of the GMZ bentonite, the CT images were analyzed using a digital volume correlation (DVC) software by Correlated Solutions. DVC is a quantitative image analysis technique for measuring internal deformation of a volume from image slices acquired via an $\mathrm{X}$-ray or CT scanner. The principle of DVC is similar to that of two-dimensional and three-dimensional Digital Image Correlation (DIC), except the $z$ dimension (through the material) is added allowing for full characterization of the material. The principle of the DIC method consists of tracking the grey-level distributions in subsets of images and pairing the homologous subsets between the reference image and the deformed images by optimizing a correlation coefficient that measures the similarity of the grey-level distribution in a small domain around these points $[19,22]$. To achieve this measurement, the DVC algorithm tracks displacement information from small volumes called voxels, similar pixel subset tracking in 2D and 3D DIC analysis. Note that image matching is performed to locate subvolumes in a series of images with optimal accuracy, and the detailed algorithm is presented in $[18,21]$. The voxel matching is performed through minimization or 


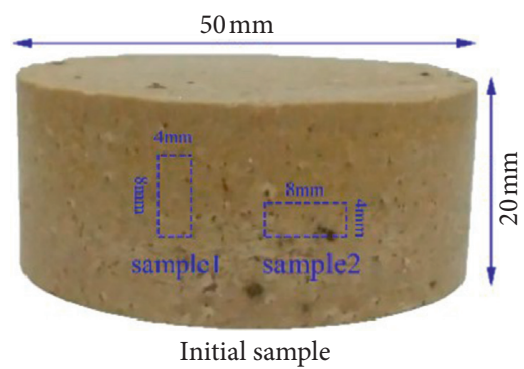

(a)

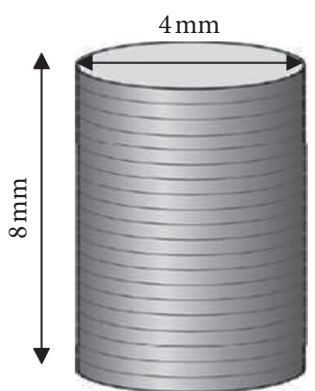

(b)

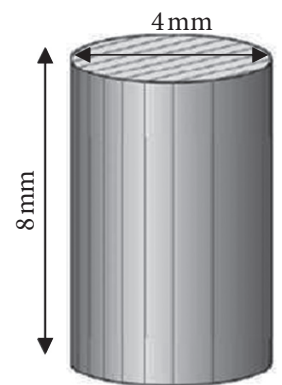

(c)

FIgURE 1: Illustration of the prepared GMZ bentonite samples: (a) photo of the specimen, (b) sample No. 1, and (c) sample No. 2.

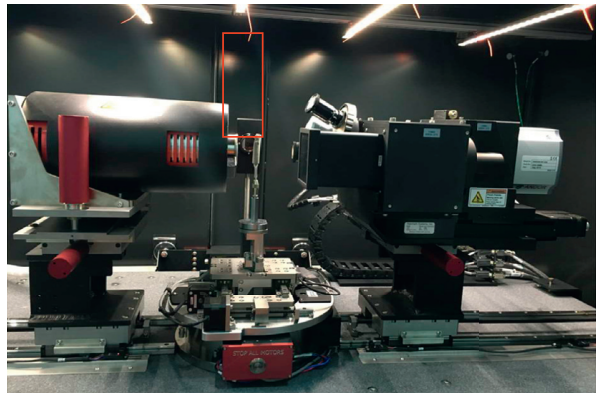

(a)

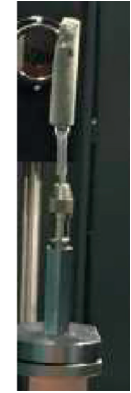

(b)

Figure 2: Photo of the micro-CT machine (a) and sample in the tube (b).

maximization of a metric function that quantifies the level of similarity in subvolumes between the reference and deformed volumetric images. The result is a $3 \mathrm{D}$ volumetric data set containing the selected strain tensor variables. The used software can determine the volumetric strains of the GMZ bentonite during drying and wetting. Note that the $z$-axis is set to be parallel to the height of the sample and the $x-y$ plane to be perpendicular to the $z$-axis, as shown in Figure 2.

\section{Results and Discussion}

The X-CT scanning of the material shows that the microstructure of the compacted GMZ bentonite is complex. It is composed of different minerals and several large grains imbed in the matrix containing fine particles, and several fissures are visible, as shown in Figure 3. Several zones (e.g., existing fissures) in the recorded images have the same grey level, and they cannot be identified through DVC as the correlated position in the deformed images cannot be found. To accurately quantify the shrinking and swelling of the GMZ bentonite, DVC has been used to analyze different zones with the size of $210 \times 210 \times 300$ voxels in the samples No. 1 and No. 2. The length of the correlating subset for DVC analysis in this study is $0.125 \mathrm{~mm}$.

\subsection{Evolution of Strains during Dehydration and Hydration.} The global strains (i.e., presenting the length of $1.05 \mathrm{~mm}$ ) of samples No. 1 and No. 2 are illustrated in Figures 4(a) and 4(b), respectively. The results show that the strains of the GMZ bentonite samples increase as the relative humidity increases and the strains decrease as the relative humidity decreases. This indicates that the GMZ bentonite compacts during drying and swells during wetting. For the sample No. 1 , the hydrated deformations in the plane perpendicular to the compaction direction (i.e., $\varepsilon_{\mathrm{xx}}$ and $\varepsilon_{\mathrm{yy}}$ ) are very similar, and they are less than $\varepsilon_{\mathrm{zz}}$ (the deformation in the direction parallel to the compaction). The results show that $\varepsilon_{\mathrm{xx}}$ and $\varepsilon_{\mathrm{yy}}$ of the sample No. 1 are close to $\varepsilon_{\mathrm{yy}}$ and $\varepsilon_{\mathrm{zz}}$ of the sample No. 2. For example, when the relative humidity decreases from $98 \%$ to $32 \%, \varepsilon_{\mathrm{zz}}$ reduces to $-5.69 \%, \varepsilon_{\mathrm{xx}}$ reduces to $-4.9 \%$, and $\varepsilon_{\mathrm{yy}}$ reduces to $-5.0 \%$ for sample No. 1 , and $\varepsilon_{\mathrm{xx}}$ reduces to $-5.55 \%$, $\varepsilon_{\mathrm{yy}}$ reduces to $-4.70 \%$, and $\varepsilon_{\mathrm{zz}}$ reduces to $-4.79 \%$ for sample No. 2 (Figures 3(a) and 3(b)).

The obtained strains $\left(\varepsilon_{\mathrm{xx}}, \varepsilon_{\mathrm{yy}}\right.$, and $\left.\varepsilon_{\mathrm{zz}}\right)$ are nonlinear and irreversible during drying and wetting, as shown in Figures 4(a) and 4(b). The nonlinear behaviour becomes more notable at higher relative humidity (e.g., $\mathrm{RH}=98 \%$ ). The mechanism of the nonlinear and irreversible behaviour is not the main topic of this paper, so it will not be discussed in detail. Several researchers have obtained similar results $[13,23,24]$. The strains induced during wetting, with $\mathrm{RH}$ increasing from $60 \%$ to $78 \%$, appear to be much larger than those during drying, when $\mathrm{RH}$ decreases from $78 \%$ to $60 \%$. This phenomenon is different from the irreversible shrinking found when one cycle of drying and wetting completes (Figure 4). In fact, during drying and wetting, the irreversible deformation is consistent with the water content curve that 


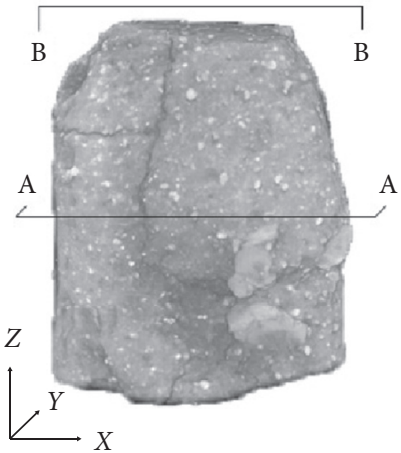

(a)

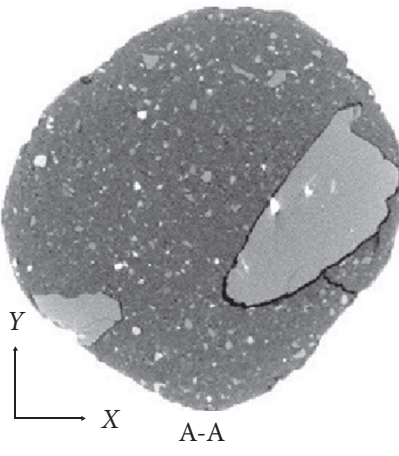

(b)

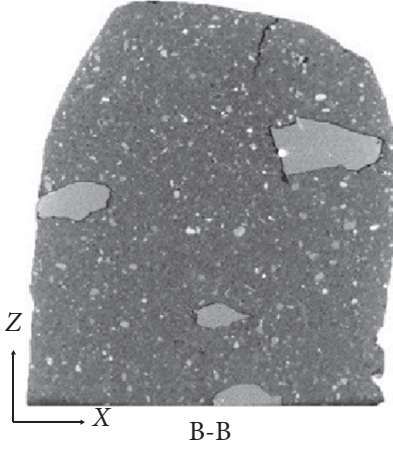

(c)

FIGURE 3: Images of the compacted GMZ bentonite: (a) 3D volume image; (b) 2D section in the $X-Y$ plane; (c) $2 \mathrm{D}$ section in the $X$ - $Z$ plane.

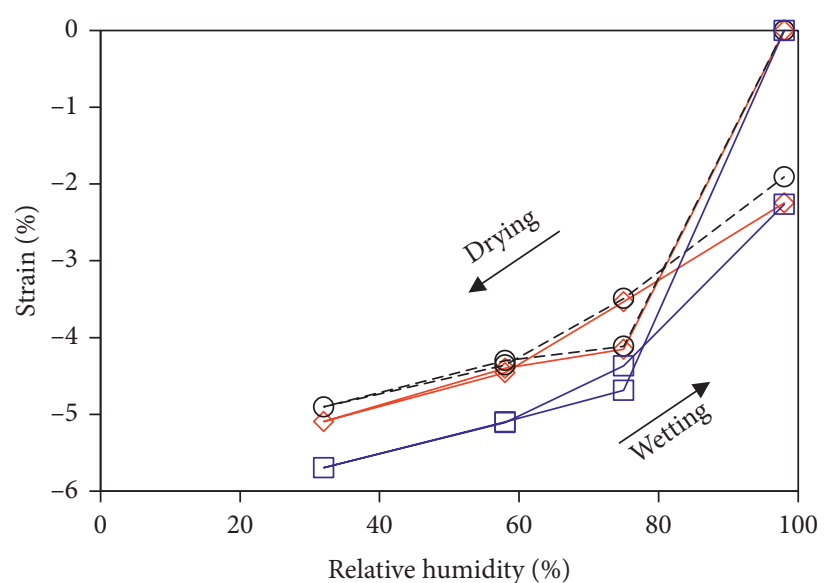

$\diamond$ Strain_xx

$-\Theta-$ Strain_yy

モStrain_zZ

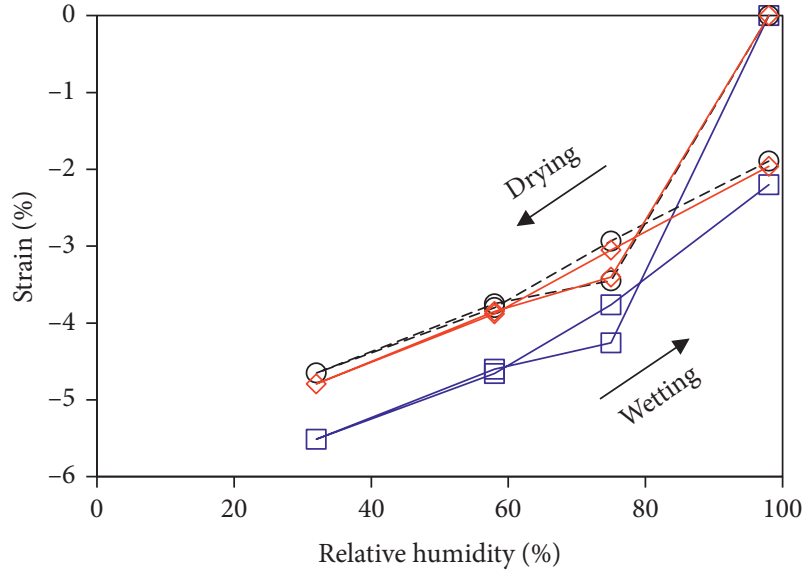

$\square$ Strain_xx

$-\Theta-$ Strain_yy

$\diamond$ Strain_zz

(b)

(a)

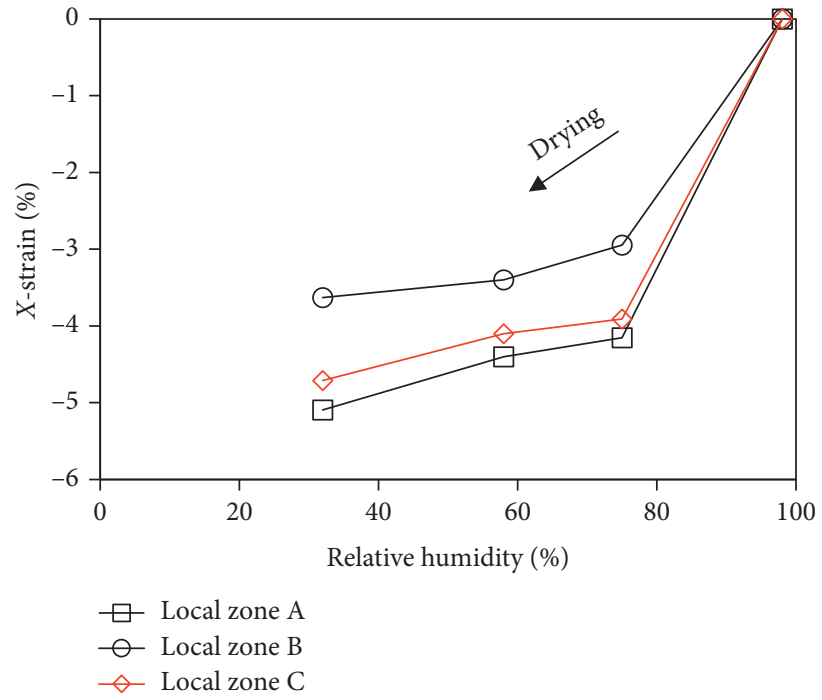

(c)

Figure 4: Strain versus relative humidity curves during drying and wetting: (a) sample No. 1; (b) sample No. 2; (c) three zones of the sample No. 1 (“-” means contraction). 


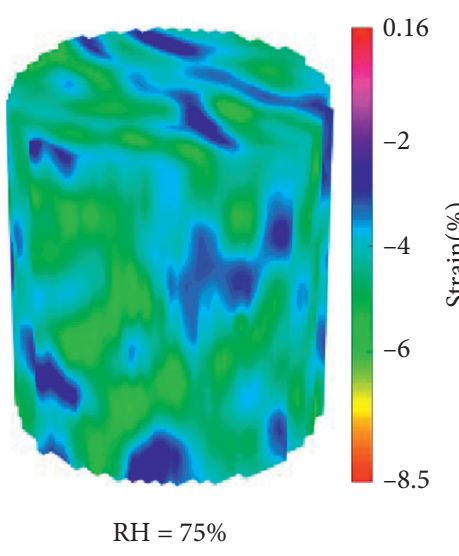

(a)

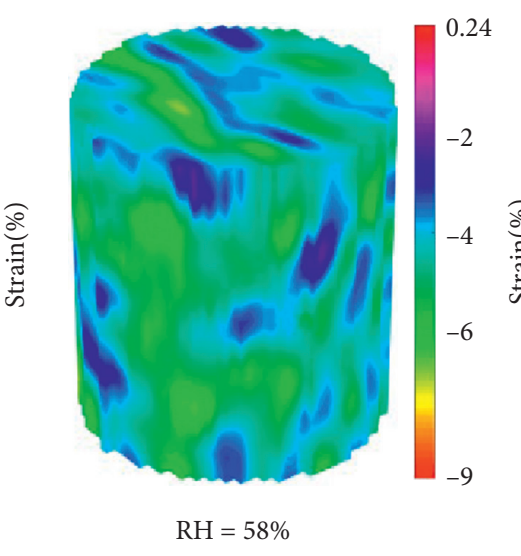

(b)

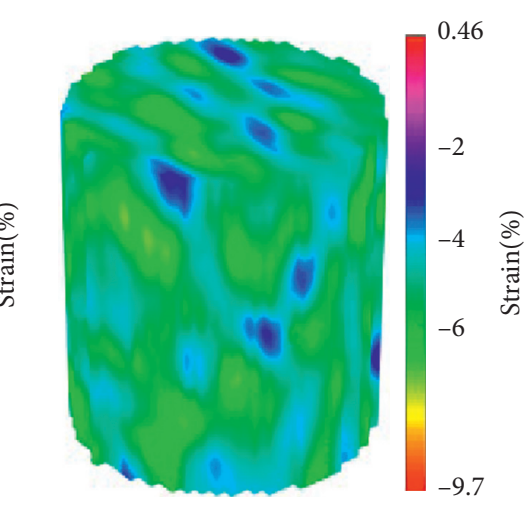

$\mathrm{RH}=32 \%$

(c)

Figure 5: Strain $\left(\varepsilon_{\mathrm{xx}}\right)$ in sample No. 1 during dehydration: (a) from $98 \%$ to $75 \%$; (b) from $98 \%$ to $58 \%$; (c) from $98 \%$ to $32 \%$ (“+” means dilatation and "-" means contraction).

exhibits a hysteresis phenomenon [14]: the low water content results in small swelling of the material. Therefore, the large swelling should be related to the high water content obtained during this wetting step.

Using DVC technique, not only the global strain of the material but also the local strain (i.e., presenting the length of $0.125 \mathrm{~mm}$ ) can be quantified. In this study, the local strain represents an equivalent gauge length of $125 \mu \mathrm{m}$, i.e., 25 voxels. Thus, the full-field strains of the GMZ bentonite during drying and wetting are obtained, and they can provide evidence of the effect of the microstructure on the shrinking and swelling of the material, as shown in Figure 5. The strain (i.e., $\varepsilon_{\mathrm{xx}}$ ) distribution of the sample No. 1 during drying is heterogeneous, and it is similar at different hydric states. Localization of the strain is notable, and it should be related to the heterogeneous microstructure. The maximal compaction strain reaches up to $8 \%$ when relative humidity decreases from $98 \%$ to $32 \%$. To further investigate the local strain, two sections of the sample No. 1 are chosen. The images of these two sections at the initial state and the measured full-field strains at different hydric states are illustrated in Figure 6. The hydric strain is mainly related to the clay minerals, while the grain is not sensible to water. The large strain in clayey matrix and small strain of the grains in Figure 6 confirm it. Moreover, localization of the strain is around the grains embed in the clay matrix, and the tensile strain around the grains indicates that tensile stress occurs at the interface between the grains and the matrix during drying. It is the cause of the appearance of new cracks around the grains.

\subsection{Anisotropy and Heterogeneity of the GMZ Bentonite.} The measured strains show that the compacted GMZ bentonite exhibits notable anisotropic shrinking-swelling properties; moreover, the shrinking-swelling deformation along the compaction direction is larger than that in the plane perpendicular to the compaction direction. The anisotropic properties could be related to several factors, e.g., the anisotropic structure or anisotropic swelling of clay minerals. As the specimen is compacted in a rigid oedometer, its fabric should be similar to sedimentary material, which has an anisotropic structure. At the same time, the strains in the plane perpendicular to the compaction direction are isotropic at the macroscopic scale. Although a few researchers confirm that the clay particle exhibits anisotropic fabric and induces anisotropic swelling [24], the observed anisotropic swelling properties (Figures 4(a) and $4(b))$ should be related to the anisotropic microstructure of the compacted GMZ bentonite.

In fact, the compacted GMZ bentonite is a heterogeneous material, and it contains a few grains, as shown in Figure 3. Thus, several zones sized $210 \times 210 \times 300$ voxels with different grain contents in the sample No.1 were chosen and analyzed; the obtained average strains $\left(\varepsilon_{\mathrm{xx}}\right)$ of the zones are illustrated in Figure 4(c). The results show that high content of grain degrades the swelling properties, confirming that the swelling properties are firmly related to clay minerals.

\subsection{Evolution of Microstructure during Dehydration and} Hydration. To clearly present the evolution of the microstructure of the compacted GMZ bentonite during drying and wetting, a representative section is chosen, and its microstructure is illustrated in Figure 7. Two distinct phenomena are found: fissures around the interface between the large grain and the matrix close during drying and reopen during wetting; several new cracks generate and propagate during drying and reclose during wetting. They should be related to different mechanisms. As shown in Figure 6, the strains in the samples are heterogeneous. As indicated above, the contribution of the matrix and inclusions to the swelling properties during wetting is different as found by several researchers $[24,25]$. The numerical study of Wang in 2014 [25] shows that the inclusion-matrix interaction results in tensile stress in the inclusion during wetting and compression stress during drying. The radial tensile stress during wetting may lead to interface separation or tangential microcracking in the matrix, whereas the tangential tensile 


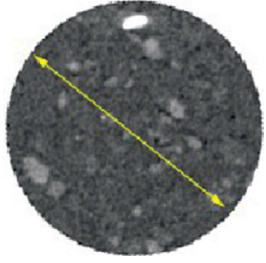

$\varphi=805.6 \mu \mathrm{m}$

(a)

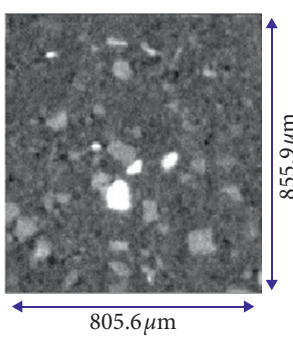

(e)

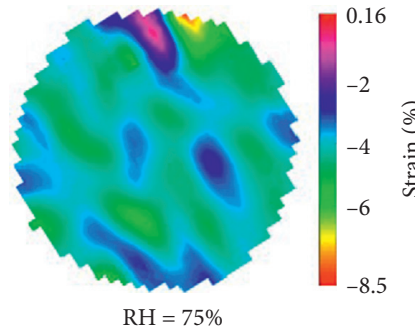

(b)

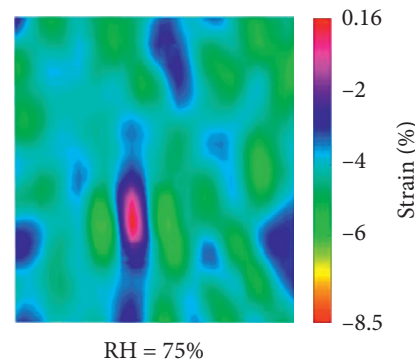

(f)

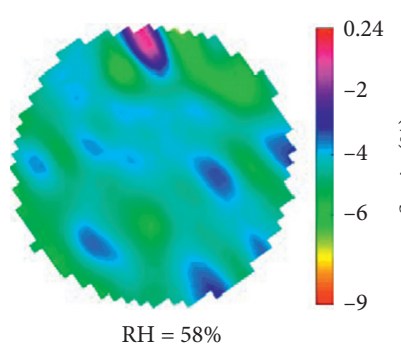

(c)

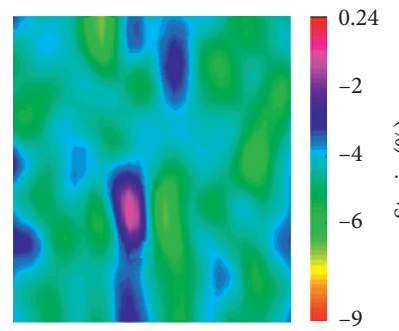

$\mathrm{RH}=58 \%$

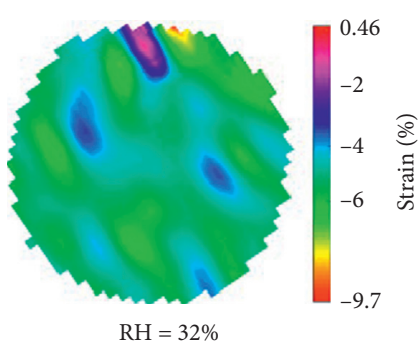

(d)

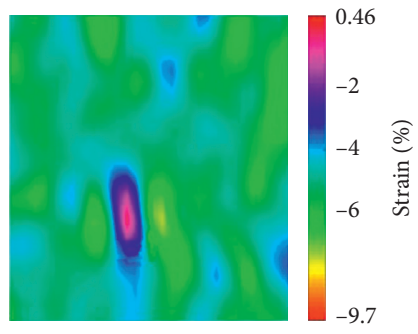

$\mathrm{RH}=32 \%$

(h)

FIgURE 6: Strains $\left(\varepsilon_{\mathrm{xx}}\right)$ in sections A-A and B-B during drying: (“+” means dilatation and “-” means contraction),

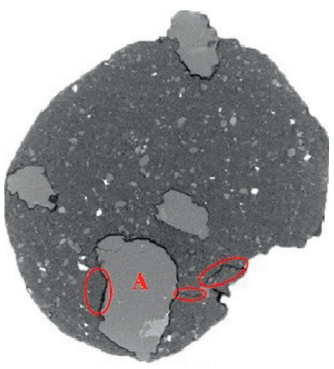

(a)

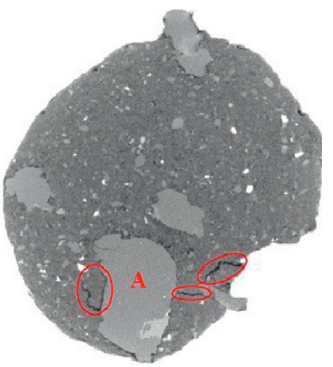

(b)

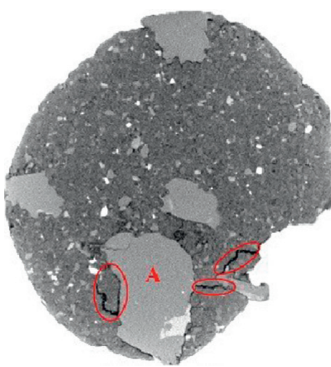

(c)

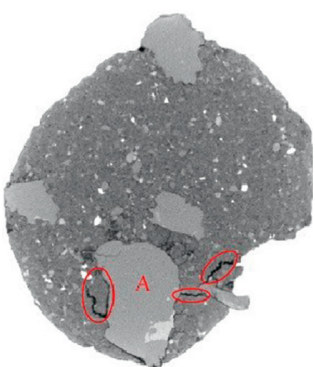

(d)

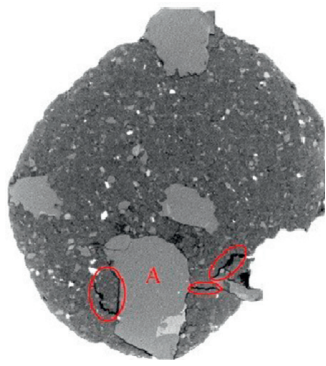

(e)

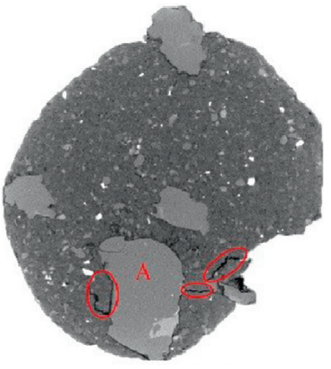

(f)

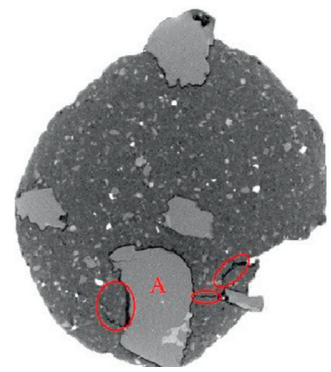

(g)

Figure 7: CT images of the GMZ bentonite during drying (from Figures 6(a) to 6(d)) and wetting (from Figures 6(d) to 6(g)): (a) RH=98\%, (b) $\mathrm{RH}=75 \%$, (c) $\mathrm{RH}=58 \%$, (d) $\mathrm{RH}=32 \%$, (e) $\mathrm{RH}=58 \%$, (f) $\mathrm{RH}=75 \%$, and (g) $\mathrm{RH}=98 \%$.

stress during drying potentially results in radial microcracking in the matrix. These numerical results are consistent with the reopening of cracks around the inclusion "A" during wetting, as shown in Figure 7. For the pre-existing fissures in the matrix, the swelling of the matrix will lead to the closure of the fissures during drying. At the same time, due to the generated tangential tensile stress, several cracks are generated in the clay matrix. Therefore, the swelling mechanism of the GMZ bentonite is complex and strongly depends on the microstructure.

\section{Conclusions}

This paper presents an experimental study on the swelling of the compacted GMZ bentonite using a technique that combined X-CT measurement with digital volume 
correlation. The prepared GMZ samples were placed in a container with constant relative humidity to reach a hydric equilibrium state and then were scanned with X-CT. The microstructure of the GMZ samples at each state during a drying and wetting cycle was recorded and analyzed using DVC. The strains in the samples during wetting and drying were quantified. The results show that the GMZ bentonite compacts during drying and swells during wetting; the maximal deformation at the macroscopic scale (i.e., in the order of $\mathrm{mm}$ ) is $5.69 \%$ when RH decreases from $98 \%$ to $32 \%$. The deformation exhibits notable anisotropic and irreversible properties during drying and wetting, and the anisotropic deformations are firmly related to the compaction direction during specimen preparation. The full-field strains in $3 \mathrm{D}$ of the materials are heterogeneous, and the local strain reaches up to $8 \%$ in the samples. The heterogeneous deformation is firmly related to the complex microstructure, and the incompatible strains of different minerals result in an internal stress and induce the appearance of cracks. The investigation of the CT images shows that there are two distinct swelling mechanisms related to the microstructure: traction occurs in the interface between the matrix and inclusions, and pre-existing fissures close during wetting. The evolution of the cracks in the GMZ bentonite could affect the performance of engineered barriers, and it should be taken into account in the design of the disposal of highlevel radioactive waste.

\section{Data Availability}

The data used are included in the article.

\section{Conflicts of Interest}

The authors declare no conflicts of interest.

\section{Authors' Contributions}

D.Yang designed the experiments; L.Chen and $\mathrm{K}$. Li carried out the tests and analyzed data. All authors have read and agreed to the published version of the manuscript.

\section{Acknowledgments}

The authors gratefully acknowledge the support of CAS Interdisciplinary Innovation Team (JCTD-2018-17), the Key Research Program of the Chinese Academy of Sciences (Grant no. KFZD-SW-423), and Hubei Provincial Natural Science Foundation of China (Grant no. 2018CFA012).

\section{References}

[1] Y. Liu, G. Xu, and S. Liu, Study on the Basic Property of Gaomiaozi Bentonite, Inner Mongolia, China Nuclear Industry Audio and Visual Publishing House, Beijing, China, 2001.

[2] W. Ye, Y. Zhang, and B. Chen, "Shear strength of an unsaturated weakly expansive soil," Journal of Rock Mechanics and Geotechnical Engineering, vol. 2, no. 2, pp. 155-161, 2010.

[3] J.-F. Liu, H.-Y. Ni, X.-L. Cao, L.-K. Ma, J.-N. Guo, and $\mathrm{X}$. Chen, "Laboratory investigation on gas permeability of compacted GMZ bentonite under a coupled hydraulic-mechanical effect," Engineering Geology, vol. 276, Article ID 105761, 2020.

[4] J. Liu, S. Song, X. Cao et al., "Determination of full-scale pore size distribution of Gaomiaozi bentonite and its permeability prediction," Journal of Rock Mechanics and Geotechnical Engineering, vol. 12, no. 2, pp. 403-413, 2020.

[5] C.-F. Tsang, F. Bernier, and C. Davies, "Geohydromechanical processes in the Excavation damaged zone in crystalline rock, rock salt, and indurated and plastic clays-in the context of radioactive waste disposal," International Journal of Rock Mechanics and Mining Sciences, vol. 42, no. 1, pp. 109-125, 2005.

[6] D. Yang, M. Bornert, S. Chanchole, H. Gharbi, and P. Valli, "Dependence on moisture content of elastic properties of argillaceous rocks investigated with optical full-field strain measurement techniques," International Journal of Rock Mechanics and Mining Sciences, vol. 54, pp. 53-62, 2012.

[7] D. Yang, S. Chanchole, and H. Gharbi, "A specific setup to study hydration and dehydration of COx argillite at applied stresses," Géotechnique Letters, vol. 4, no. 2, pp. 94-97, 2014.

[8] W. M. Ye, N. C. Borrell, J. Y. Zhu, B. Chen, and Y. G. Chen, "Advances on the investigation of the hydraulic behavior of compacted GMZ bentonite," Engineering Geology, vol. 169, pp. 41-49, 2014.

[9] C. Amorim, R. Lopes, R. Barroso, J. Queiroz, D. Alves, and C. Perez, "Effect of clay-water interactions on clay swelling by X-ray diffraction," Nuclear Instruments and Methods in Physics Research Section A, vol. 580, no. 1, pp. 768-770, 2007.

[10] R. Viola, M. Tuller, D. Or, and J. Drasdis, "Microstructure of clay-sand mixtures at different hydration states," in Advanced Experimental Unsaturated Soil Mechanics, A. Tarantino, E. Romero, and Y. Cui, Eds., pp. 437-442, Taylor, Francis Group, London, UK, 2005.

[11] P. Delage, M. D. Howat, and Y. J. Cui, "The relationship between suction and swelling properties in a heavily compacted unsaturated clay," Engineering Geology, vol. 50, no. 1-2, pp. 31-48, 1998.

[12] L. Chen, H. Peng, and D. Yang, "The microstructure and water distribution of partially saturated hard clay," Geotechnical Testing Journal, vol. 41, no. 4, pp. 830-835, 2018.

[13] G. Montes-H, J. Duplay, L. Martinez, and C. Mendoza, "Swelling-shrinkage kinetics of MX80 bentonite," Applied Clay Science, vol. 22, no. 6, pp. 279-293, 2003.

[14] J. Mitchell and K. Soga, Fundamentals of Soil Behavior, John Wiley and Sons, Inc, New Jersey, NY, USA, Third edition, 2005.

[15] E. Romero and P. Simms, "Microstructure investigation in unsaturated soils: a review with special attention to contribution of mercury intrusion porosimetry and environmental scanning electron microscopy," Geotechnical and Geological Engineering, vol. 26, no. 6, pp. 705-728, 2008.

[16] E. Ando, G. Viggiani, S. Hall, and J. Desrues, "Experimental micro-mechanics of granular media studies by X-ray tomography: recent results and challenges," Géotechnique Letters, vol. 3, no. 3, pp. 142-146, 2013.

[17] J. Bruchon, J. Pereira, M. Vandamme, N. Lenoir, P. Delage, and M. Bornert, "X-ray microtomography characterisation of the changes in statistical homogeneity of an unsaturated sand during imbibition," Géotechnique Letters, vol. 3, no. 2, pp. 84-88, 2013.

[18] R. Brault, A. Germaneau, J. C. Dupré, P. Doumalin, S. Mistou, and M. Fazzini, "In-situ analysis of laminated composite materials by X-ray micro-computed tomography and digital 
volume correlation," Experimental Mechanics, vol. 53, no. 7, pp. 1143-1151, 2013.

[19] N. Lenoir, M. Bornert, J. Desrues, P. Bésuelle, and G. Viggiani, "Volumetric digital image correlation applied to X-ray microtomography images from triaxial compression tests on argillaceous rock," Strain, vol. 43, no. 3, pp. 193-205, 2007.

[20] S. Hall, M. Bornert, J. Desrues et al., "Discrete and continuum analysis of localised deformation in sand using X-ray Micro CT and volumetric digital image correlation," Geotechnique, vol. 60 , no. 5, pp. 11-20, 2010.

[21] S. Lee and W. Ji, "DVC analysis of a polymer material subjected to tensile loading with synchrotron radiation tomography," Polymer Testing, vol. 81, Article ID 106204, 2020.

[22] M. Sutton, J. Orteu, and H. Schreier, Image Correlation for Shape, Motion and Deformation Measurements: Basic Concepts, Theory and Applications, Springer, Berlin, Germany, 2009.

[23] A. Gens and E. E. Alonso, "A framework for the behaviour of unsaturated expansive clays," Canadian Geotechnical Journal, vol. 29, no. 6, pp. 1013-1032, 1992.

[24] L. L. Wang, M. Bornert, E. Héripré, D. S. Yang, and S. Chanchole, "Irreversible deformation and damage in argillaceous rocks induced by wetting/drying," Journal of Applied Geophysics, vol. 107, pp. 108-118, 2014.

[25] L. Wang, A. Pouya, B. Halphen, and M. Bornert, "Modeling the internal stress filed in argillaceous rocks under humidification/desiccation," International Journal for Numerical and Analytical Methods in Geomechanics, vol. 38, no. 16, pp. 1664-1682, 2014. 